hep-th/9512024, RU-95-91

\title{
A Comment on Non-Chiral Operators in SQCD and its Dual
}

\author{
M. Berkooz * \\ Department of Physics and Astronomy \\ Rutgers University \\ Piscataway, NJ 08855-0849
}

\begin{abstract}
We match a few non chiral operators in the electric and magnetic descriptions of SQCD, suggesting the first evidence of electric-magnetic duality outside the chiral ring. Algebraically, these non chiral operators are a module of the chiral ring. Physically, the suggested correspondence essentially transforms certain electric gauge invariant composites containing the electric field strength into magnetic matter composites.
\end{abstract}

* e-mail: berkooz@physics.rutgers.edu 


\section{Introduction}

There are two sets of operators whose status in $\mathrm{N}=1$ electric-magnetic duality [1] (for a recent review see [2]) is unclear. The first are non chiral operators and the second are chiral operators of the form, in say SQCD, $\widetilde{Q}^{T} W_{\alpha} W_{\beta} \ldots Q$. The first class constitutes most of the operators in the theory and their mapping under duality is important if one really wishes to compute a physical process in the dual theory. These operators, however, are under much less control then chiral operators and their transformation under duality is unclear, leaving the nagging, albeit implausible, worry that this is not an equivalence of the full theory. The second class are trivially included in the first class as they are their descendants. (for example, $\widetilde{Q}^{T} W_{\alpha} Q$ is obtained from $\widetilde{Q}^{T} \mathbf{D}_{\alpha} Q$ (where $\mathbf{D}_{\alpha}$ is the covariant derivative in superspace) by applying $\bar{D}^{2}$, and rewriting the constrained super-connection in terms of $W_{\alpha}$ ). They are, however, of immediate relevance since they are a gauge invariant way of "measuring" the field strength operator in either theory.

In the following we will partially identify a small set of non-chiral operators. Some of the identifications are possible due to degeneracies in the conformal representation of these operators, allowing the calculation of their conformal dimension (the identification is up to a certain well defined ambiguity).

The identification is the simplest when assuming a canonical Kähler potential, but is valid for an arbitrary one. In the general case one sees that the exchanged operators correspond to geometrical objects on the two corresponding Kähler manifolds.

In section 2 we discuss some gauge fixing conventions, most of which are already in the literature, and some new ways of handling them concisely. Section 3 discusses the actual correspondence. Section 4 contains a discussion of the correspondence for SQCD with the addition of a matter field in the adjoint representation. Section 5 discusses the generalization to an arbitrary Kähler potential. We conclude with a brief observation regarding the gauge field strength in the theories.

\section{Notation and Equations of Motion}

We do not really have control over the non-chiral objects in a strongly coupled super- 
symmetric field theory. But, following examples from two dimensional field theories, there are still interesting non-chiral objects one can study.

The main example in two dimensional field theory is the correspondence between Landau-Ginzburg theories and minimal models, which was suggested in [3] for non $\mathrm{Su}$ persymmetric theories (and thus has nothing to do with chirality). We will therefore be interested in essentially formal strings of the allowed symbols at hand, i.e. fields and covariant derivatives, modulo some relations. The relations are kinematical, such as the constraints in the curvature tensor, and dynamical, such as the equations of motion. Admittedly, this approach is not rigorously justified and in fact can be shown to be wrong in some cases (for a detailed analysis of an example see [7]) but in this case it yields correct results as one can see from assessing the quantum corrections to the formula, at least when the theory is taken to weak coupling. One can also compare it with results from the representation theory of the superconformal algebra.

It is convenient to work in a covariant formulation [4] [6] in which gauge transformations are taken to be maps from superspace into unitary operators rather then maps of the form $e^{i \Lambda}$, where $\Lambda$ is a Lie algebra valued chiral superfield, which is the more commonly encountered gauge.

More precisely, gauge transformations are given by elements in the "group", i.e. $e^{i K^{i} T_{i}}$ where $K$ are real superfields (and so the lowest component of this transformation is a usual gauge transformation). One also introduces a superspace gauge connection, which is restricted by the equations

$$
F_{\alpha \beta}=F_{\dot{\alpha} \dot{\beta}}=F_{\alpha \dot{\beta}}=0
$$

where $F$ is the superspace curvature form associated with the connection (these equations are made more explicit below). One can pick the solution to the constraints on the curvature form to be $\overline{\mathbf{D}}_{\dot{\alpha}}=e^{-\bar{\Omega}} D_{\dot{\alpha}} e^{\bar{\Omega}}$ and $\mathbf{D}_{\alpha}=e^{-\Omega} D_{\alpha} e^{\Omega}$ where $D$ is the familiar superspace covariant derivative and $\mathbf{D}$ is the gauged covariant derivative (under an infinitesimal gauge transformation the fields transform according to $\delta \Omega \rightarrow-i K+\bar{\Lambda}, \delta \bar{\Omega} \rightarrow-i K+\Lambda$ ). To pass to the usual formulation one can now choose a gauge in which $\Omega=-\bar{\Omega}$, and the usual gauge field is $e^{V}=e^{\Omega} e^{-\bar{\Omega}}$.

Chiral fields in this formulation are defined as $\overline{\mathbf{D}}_{\dot{\alpha}} \phi=0$ and are related to the usual 
definition by multiplying by appropriate powers of $e^{\bar{\Omega}}$, and similarly antichiral fields will be related to the usual definition by $e^{\Omega}$. From a more practical point of view the formulas we will write below in this gauge will be related to formulas in the usual gauge essentially by inserting $e^{V}$ whenever necessary, for example $Q^{\dagger} e^{V} Q$ in the usual notation will now be just $Q^{\dagger} Q$.

The constraints on the classical chain of symbols are kinematical and dynamical. The kinematical constraints $(2.1)$ on the curvature tensor are

$$
\left\{\mathbf{D}_{\alpha}, \mathbf{D}_{\beta}\right\}=\left\{\overline{\mathbf{D}}_{\dot{\alpha}}, \overline{\mathbf{D}}_{\dot{\beta}}\right\}=0, \quad \mathbf{D}_{\mu} \propto\left\{\mathbf{D}_{\alpha}, \overline{\mathbf{D}}_{\dot{\beta}}\right\}
$$

We will take the third one into account just by always writing $\mathbf{D}_{\mu}$ in terms of $\mathbf{D}_{\alpha}, \overline{\mathbf{D}}_{\dot{\alpha}}$.

The dynamical constraints are the equations of motion. These are the F-term equations

$$
D^{2} Q=-4 \frac{\partial W^{*}}{\partial Q^{*}}, \bar{D}^{2} Q^{*}=-4 \frac{\partial W}{\partial Q}
$$

and the D-term equations, which for an $S U\left(N_{c}\right)$ theory with $Q(\widetilde{Q})$ fields in the (anti) fundamental rep. are

$$
\frac{1}{g^{2}} D^{\alpha} W_{\alpha}=Q_{i} Q_{i}^{\dagger}-\widetilde{Q}_{\tilde{i}}^{*} \widetilde{Q}_{\tilde{i}}^{T}-\frac{I_{N_{c} \times N_{c}}}{N_{c}}\left(Q_{i}^{\dagger} Q_{i}-\widetilde{Q}_{\tilde{i}}^{T} \widetilde{Q}_{\tilde{i}}^{*}\right)
$$

rewriting the field strength superfield $W_{\alpha}$ in terms of the curvature form [4] [5], we obtain, in our notation, the relation

$$
\begin{gathered}
\frac{1}{g^{2}} \epsilon^{\beta \gamma} \epsilon^{\dot{\delta} \dot{\beta}}\left(\mathbf{D}_{\gamma} \mathbf{D}_{\beta} \overline{\mathbf{D}}_{\dot{\beta}} \overline{\mathbf{D}}_{\dot{\delta}}-\overline{\mathbf{D}}_{\dot{\beta}} \overline{\mathbf{D}}_{\dot{\delta}} \mathbf{D}_{\gamma} \mathbf{D}_{\beta}+2 \mathbf{D}_{\gamma} \overline{\mathbf{D}}_{\dot{\beta}} \mathbf{D}_{\beta} \overline{\mathbf{D}}_{\dot{\delta}}-2 \overline{\mathbf{D}}_{\dot{\delta}} \mathbf{D}_{\beta} \overline{\mathbf{D}}_{\dot{\beta}} \mathbf{D}_{\gamma}\right)= \\
=Q_{i} Q_{i}^{\dagger}-\widetilde{Q}_{\tilde{i}}^{*} \widetilde{Q}_{\tilde{i}}^{T}-\frac{I_{N_{c} \times N_{c}}}{N_{c}}\left(Q_{i}^{\dagger} Q_{i}-\widetilde{Q}_{\tilde{i}}^{T} \widetilde{Q}_{\tilde{i}}^{*}\right)
\end{gathered}
$$

If one has a general Kähler form then the equations are modified and are: F-term equation:

$$
D^{2} \frac{\partial K}{\partial Q^{*}}=-4 \frac{\partial W^{*}}{\partial Q^{*}}
$$

and its conjugate.

D-term equation: we have to replace the above Yang-Mills current by an expression that takes into account the Kähler form. The general expression for a current, labeled by 
$a$, that corresponds to a symmetry generated by a vector field $v^{(a)}+v^{(a) *}$ on the Kähler manifold, is just $v^{(a)} K$. Thus in our case $\frac{1}{g^{2}} D^{\alpha} W_{\alpha}^{a}=v^{(a)} K, a$ here is an index in the adjoint of the gauge group. Both the $F$ and $D$ term equations are invariant under Kähler transformations by a gauge invariant holomorphic function.

A useful property of a symmetry current is

$$
\bar{D}^{2}\left(v^{a} K\right)=\bar{D}^{2} v^{a, i} \frac{\partial K}{\partial x^{i}}=v^{a, i} \bar{D}^{2} \frac{\partial K}{\partial x^{i}}=-4 v^{a, i} \frac{\partial W}{\partial x^{i}}=0 .
$$

Note that if we assume that the global symmetry currents are primary, i.e. $\bar{S}_{\dot{\alpha}}\left(v^{(a)} K\right)=$ 0 , then we consistently get from the superconformal algebra that they have the correct dimension 2 .

The operators that will be identified below live in degenerate representations of the superconformal algebra. Following [8][9], the representations of the $\mathrm{N}=1$ superconformal algebra are labeled by $(j, \bar{j}, D, R)$ where $j(\bar{j})$ is the left handed (right handed) $S U(2)$ representation, $D$ is the conformal dimension (normalized to 1 on free scalar fields) and $R$ is the $R$ charge (normalized to 1 on gauginos). The representations fall into three categories. Two of these categories are the chiral and anti-chiral representations, which are the representations in which (in the spinless case) the chiral (and anti-chiral) ring lives. We will be interested here in the third type of representation. This type satisfies

$$
D \geq\left|\frac{3}{2} R-j+\bar{j}\right|+j+\bar{j}+2
$$

and it becomes free when $j \bar{j} \neq 0, \frac{3}{2} R=j-\bar{j}$ and $D=j+\bar{j}+2$.

The current noted above is in this type of a representation with $j=\bar{j}=0$ and the representation is degenerate, satisfying $D=2$.

The other operators we will discuss below transform as $\left(\frac{1}{2}, 0\right)$ under the Lorentz group, their $(0,0)$ descendant is null and they satisfy $\frac{3}{2} R<\frac{1}{2}$. Thus their dimension satisfies $D=-\frac{3}{2} R+3$. If we now multiply such an operator by an anti-chiral operator, then by standard arguments [1] [10] there are no short distance singularities and result is again a degenerate $\left(\frac{1}{2}, 0\right)$ operator, with the same kind of degeneracy, satisfying $\frac{3}{2} R<\frac{1}{2}$. Thus these operators form a module of the anti-chiral ring (there is similarly a module of the chiral ring). 


\section{SQCD and its dual}

Our main purpose is to identify some non-chiral operators between the electric and the magnetic descriptions of SQCD.

For completeness, we will write down the electric and magnetic theories. The electric theory contains fundamentals and anti-fundamentals of $S U\left(n_{c}\right)$ with global charges

$\begin{array}{lllll} & S U\left(n_{F}\right) & S U\left(n_{\bar{F}}\right) & U(1)_{B} & U(1)_{R} \\ Q & n_{F} & 1 & 1 & 1-\frac{n_{c}}{n_{f}} \\ \widetilde{Q} & 1 & n_{\bar{F}} & -1 & 1-\frac{n_{c}}{n_{f}}\end{array}$

and no superpotential.

The magnetic theory contains fundamentals $(q)$ and anti-fundamentals $(\tilde{q})$ of a gauge group $S U\left(n_{F}-n_{c}\right)$ and an uncharged meson $M$, with global charges

$\begin{array}{lllll} & S U\left(n_{F}\right) & S U\left(n_{\bar{F}}\right) & U(1)_{B} & U(1)_{R} \\ q & \bar{n}_{F} & 1 & \frac{n_{c}}{n_{F}-n_{c}} & \frac{n_{c}}{n_{f}} \\ \widetilde{q} & 1 & \bar{n}_{\bar{F}} & \frac{n_{c}}{n_{F}-n_{c}} & \frac{n_{c}}{n_{f}} \\ M & n_{F} & n_{\bar{F}} & 1 & 2-\frac{2 n_{c}}{n_{f}}\end{array}$

The superpotential in the magnetic theory is $c q \bar{q} M$ and $M$ is identified with the electric composite $Q \bar{Q}$.

In this section we will take the Kähler potential in both theories to be of the canonical form.

The most immediate identifications are those of the global symmetry currents. Generally:

$$
v_{e}^{(a)} K_{e} \leftrightarrow v_{m}^{(a)} K_{m}
$$

for any symmetry with a vector field generator $v^{(a)}$ on the Kähler manifold. The argument for these identifications is that the global symmetry current is a unique object and it does not matter which variables we use to describe it.

In our case:

$\begin{array}{lll}\text { Current } & \text { Electric } & \text { Magnetic } \\ S U\left(n_{F}\right) & P_{T}\left(Q^{i T} Q_{j}^{*}\right) & P_{T}\left(-q_{j}^{T} q^{i *}+M^{i \tilde{k}} M_{j \tilde{k}}^{*}\right) \\ S U\left(n_{\tilde{F}}\right) & P_{T}\left(\widetilde{Q}^{i T} \widetilde{Q}_{\tilde{j}}^{*}\right) & P_{T}\left(-\tilde{q}_{\tilde{j}}^{T} \tilde{q}^{\tilde{i *}}+M^{k \tilde{i}} M_{\tilde{k}_{\tilde{j}}}^{*}\right) \\ U(1)_{B} & Q^{i T} Q_{i}^{*}-\widetilde{Q}^{\tilde{i T}} \widetilde{Q}_{\tilde{i}} & \frac{n_{c}}{n_{f}-n_{c}}\left(q_{i}^{T} q^{i *}-\tilde{q}_{\tilde{i}}^{T} \tilde{q}^{\tilde{i} *}\right)\end{array}$


where $P_{T}$ is a projection on traceless matrices. We will denote these currents by $T, \widetilde{T}$ and $T_{B}$

The two other identifications we would like to show are:

Electric

Magnetic

$$
\begin{aligned}
\widetilde{Q}_{\tilde{k}}^{T} \mathbf{D}_{\alpha} Q_{i}- & \left(\mathbf{D}_{\alpha} \widetilde{Q}_{\tilde{k}}\right)^{T} Q_{i} \leftrightarrow \epsilon^{\dot{\alpha} \dot{\beta}}\left(\tilde{q}_{\tilde{k}}^{\dagger} \overline{\mathbf{D}}_{\dot{\alpha}} \mathbf{D}_{\alpha} \overline{\mathbf{D}}_{\dot{\beta}} q_{l}^{*}\right)+\text { products }+ \text { descendant } \\
\epsilon^{\dot{\alpha} \dot{\beta}} \widetilde{Q}_{\tilde{k}}^{\dagger} \overline{\mathbf{D}}_{\dot{\alpha}} \mathbf{D}_{\alpha} \overline{\mathbf{D}}_{\dot{\beta}} Q_{i}^{*}+(\widetilde{Q} \leftrightarrow Q) & \leftrightarrow \tilde{q}_{\tilde{k}}^{T} \mathbf{D}_{\alpha} q_{l}-\left(\mathbf{D}_{\alpha} \tilde{q}_{\tilde{k}}\right)^{T} q_{l}
\end{aligned}
$$

These four operators are in a $\left(\frac{1}{2}, 0\right)$ representation of the Lorentz group. They have the property that, using the $D$ or the $F$ equations of motion, their $(0,0)$ descendant is either a product of simpler operators or is null, and thus can be identified in the dual. We see that duality interchanges factorization via the $F$ equation of motion and via the $D$ equation of motion. Of course, the identification is up to operators that have null $(0,0)$ descendant, a point to which we will return later.

To show the equivalence, one proceeds as follows. Since one of the theories in question is strongly coupled, we can not really do any equivalent computation in both theories. What we will do, in the spirit explained above, is a classical calculation and then try to check whether it is valid quantum mechanically.

We begin with (3.2). The exact correspondence is:

$$
O^{e}=\widetilde{Q}_{\tilde{k}}^{T} \mathbf{D}_{\alpha} Q_{l}-\left(\mathbf{D}_{\alpha} \tilde{Q}_{\tilde{k}}\right)^{T} Q_{l}
$$

maps to

$$
\begin{aligned}
O^{m}= & \epsilon^{\dot{\alpha} \dot{\beta}}\left(\tilde{q}_{\tilde{k}}^{\dagger} \overline{\mathbf{D}}_{\dot{\alpha}} \mathbf{D}_{\alpha} \overline{\mathbf{D}}_{\dot{\beta}} q_{l}^{*}+\left(\overline{\mathbf{D}}_{\dot{\beta}} \mathbf{D}_{\alpha} \overline{\mathbf{D}}_{\dot{\alpha}} \tilde{q}_{\tilde{k}}^{*}\right)^{T} q_{l}^{*}\right)+ \\
& \left.+\frac{1}{2}\left(\left(\tilde{q}_{\tilde{k}}^{\dagger} \overline{\mathbf{D}}_{\dot{\alpha}}^{2} q_{l}^{*}\right)-\left(\overline{\mathbf{D}}_{\dot{\alpha}}^{2} \tilde{q}_{\tilde{k}}^{*}\right)^{T} q_{l}^{*}\right)\right)- \\
& -\frac{g^{2}}{2 c}\left(\mathbf{D}_{\alpha}\left(M_{s \tilde{k}} T_{l}^{s}\right)-2 M_{s \tilde{k}}\left(\mathbf{D}_{\alpha} T_{l}^{s}\right)\right. \\
& +\frac{g^{2}}{2 c}\left(\mathbf{D}_{\alpha}\left(\widetilde{T}_{\tilde{k}}^{\tilde{l}} M_{l \tilde{l}}\right)-2\left(\mathbf{D}_{\alpha} \widetilde{T}_{\tilde{k}}^{\tilde{l}}\right) M_{l \tilde{l}}\right)+ \\
+ & \frac{g^{2}}{c} \frac{n_{F}-}{n_{c}} \\
n_{c} & \left(\frac{1}{n_{f}}-\frac{1}{n_{c}}\right)\left(\mathbf{D}_{\alpha}\left(M_{l \tilde{k}} T_{B}\right)-2 M_{l \tilde{k}} \mathbf{D}_{\alpha} T_{B}\right)+
\end{aligned}
$$




$$
+\frac{g^{2}}{2 c}\left(\left(\mathbf{D}_{\alpha} M^{T}\right) M^{*} M^{T}-M^{T} M^{*} \mathbf{D}_{\alpha} M^{T}\right)
$$

And a direct classical calculation yields that the $(0,0)$ descendant of both sides vanishes (and that all global quantum numbers match).

By the general properties of the representations of the superconformal algebra we know that the conformal dimension of these operators is $\frac{3}{2}+\frac{3 N_{c}}{N_{f}}$. There are two ways in which we can go to weak coupling. We can take $N_{f} \rightarrow 3 N_{c}$ and approach weak electric coupling 13], in which case $D \rightarrow \frac{5}{2}$ which is the correct classical result for the electric operator, or we can take $N_{f} \rightarrow \frac{3}{2} N_{c}$ and approach weak magnetic coupling, in which case $D \rightarrow \frac{7}{2}$, which is the correct classical result for the magnetic operator.

The fact that in the weak coupling limit the quantum dimension converges to the classical dimension for this operator lends further credence to the assumptions going into calculating its quantum dimension, namely that it is primary and has a null descendant, and that one should look for it in the dual theory.

We can obviously always add to either sides descendants, or "products" of operators, with appropriate quantum numbers, that satisfy $D^{\alpha} O_{\alpha}=0$. We would like, however, to claim that there is no arbitrariness in the part that contains the covariant derivatives.

More precisely, we would like to show that there is no other operator of the form, say, $\widetilde{Q}_{\tilde{k}}^{T} \mathbf{D} \ldots \mathbf{D} Q_{l}$ that has the same quantum numbers as $\widetilde{Q}_{\tilde{k}}^{T} \mathbf{D}_{\alpha} Q_{l}$ and whose $(0,0) D_{\gamma}$ descendant is null. The reason is the following. If the descendant is null and we know the R-charge then we can calculate the conformal dimension of the operator to be $\frac{3}{2} R+3$. If we now take the limit $N_{c}, N_{f} \rightarrow \infty$ at a fixed ratio $\frac{N_{f}}{N_{c}} \rightarrow 3-\epsilon$ then we are in a weakly coupled theory and we can approximate the dimension of the operator by the classical dimension. In particular each $D$ contributes $\frac{1}{2}$ to the dimension. We thus have the sum and difference (from the R charge) of the number of $D$ 's and $\bar{D}$ 's and can easily see that $\widetilde{Q} D Q$ is the only allowed operator with such a degeneracy for these quantum numbers.

Similarly, one is unable to construct any other operator of the form $\tilde{q}^{\dagger} D \ldots D q+$ descendants + products with the required properties besides $\tilde{q}^{\dagger} \bar{D} D_{\alpha} \bar{D} q$. The reasoning is similar.

Thus only these combinations of covariant derivatives have a chance of living in a 
degenerate rep. of the Superconformal algebra with $\left(\frac{1}{2}, 0\right)$ primary field, and indeed we have shown that they can be extended to a degenerate operator.

We cannot show that our manipulations hold Quantum Mechanically. However, the picture presented thus far is the simplest, if not the only, one that might work and that is self consistent.

To show (3.3), we would like to calculate the $(0,0)$ descendants of both sides. In the magnetic theory:

$$
\begin{aligned}
& \frac{-1}{4 c} \epsilon^{\rho \alpha} D_{\rho} O_{\alpha}^{m}=\tilde{q}_{\tilde{k}}^{T} \tilde{q}^{\tilde{i} *} M_{\tilde{i}}^{*}-M_{\tilde{s} k}^{*} q^{s \dagger} q_{l}= \\
& =T_{l}^{s} M_{s \tilde{k}}^{*}-\widetilde{T}_{\tilde{k}}^{\tilde{i}} M_{l \tilde{i}}^{*}-\frac{n_{f}-n_{c}}{n_{f} n_{c}} T_{B} M_{l \tilde{k}}
\end{aligned}
$$

and in the electric theory:

$$
\begin{aligned}
\epsilon^{\rho \alpha} O_{\alpha}^{e} & \propto\left(\widetilde{Q}_{\tilde{k}}^{\dagger} \widetilde{Q}_{\tilde{i}}\right)\left(\widetilde{Q}_{\tilde{i}}^{\dagger} Q_{\tilde{l}}^{*}\right)-\left(\widetilde{Q}_{k}^{\dagger} Q_{j}^{*}\right)\left(Q_{j}^{T} Q_{l}^{*}\right)-\frac{1}{n_{c}} M_{l \tilde{k}} T_{B}= \\
& =-\left(T_{l}^{s}\right) M_{s \tilde{k}}^{*}+\widetilde{T}_{\tilde{k}}^{\tilde{i}} M_{l \tilde{i}}^{*}+\left(\frac{1}{n_{c}}-\frac{1}{n_{f}}\right) T_{B} M_{l \tilde{k}}
\end{aligned}
$$

Again, these are in agreement.

\section{Another Example}

The discussion above can be repeated in other models. An interesting model has $S U\left(N_{c}\right)$ gauge group with $N_{f}$ quarks $(Q)$ and anti-quarks $(\widetilde{Q})$ and a matter field $(X)$ in the adjoint representation of the gauge group [1] [7] (and some extensions of it in [12]). We will not repeat here the details of the model.

In this model, if the electric superpotential is $\operatorname{Tr} X^{k+1}$, then one can define the degenerate electric operators $\widetilde{Q} X^{* t} \mathbf{D}_{\alpha} Q-(\widetilde{Q} \leftrightarrow Q)$ for $t<k$. These are matched with a linear combination of $\left\{\tilde{q}^{*} Y^{* s} \overline{\mathbf{D}} \cdot \mathbf{D}_{\alpha} \overline{\mathbf{D}} \cdot Y^{* k-1+t-s} q^{*}, s=1 \ldots k-1+t\right\}$ (plus some descendants and products).

It is interesting to note the following relationship between the degenerate operators that we have discussed, and the structure of the chiral ring. Suppose we have an electric operator of the form $C_{1} \mathbf{D}_{\alpha} C_{2}$ where $C_{1}$ and $C_{2}$ are chiral operators and its magnetic counterpart is $\epsilon^{\dot{\alpha} \dot{\beta}} \hat{C}_{1}^{*} \overline{\mathbf{D}}_{\dot{\alpha}} \mathbf{D}_{\alpha} \overline{\mathbf{D}}_{\dot{\beta}} \hat{C}_{2}^{*}$. It is then natural to have a term $M \hat{C}_{1} \hat{C}_{2}$ in the magnetic 
superpotential, where $M$ is a fundamental field in the magnetic theory that is identified with $C_{1} C_{2}$ of the electric theory. Thus the product $\hat{C}_{1} \hat{C}_{2}$ is zero in the chiral ring, while $\hat{C}_{1}$ and $\hat{C}_{2}$ are not zero in the chiral ring in the sense that there are non trivial chiral primaries that contain them.

This is the case for the operator $\widetilde{Q} \mathbf{D}_{\alpha} Q-\left(\mathbf{D}_{\alpha} \widetilde{Q}\right) Q$. But we can also analyze the operator $\widetilde{Q} X^{*} Q$, in say the $\mathrm{k}=2$ model. The corresponding anti-chiral operator, after dropping the covariant derivatives, is $\tilde{q}^{*} Y^{* 2} q^{*}$. This operator can be simplified by the equation of motion coming from $W_{m a g}=\operatorname{Tr} Y^{3}$, and is thus a sum of products of simpler chiral primaries. Note that this operator could not have been eliminated from the chiral ring by coupling it, in the superpotential,

to a magnetic gauge invariant field. The operator it can couple to from the electric theory is $\widetilde{Q} X^{*} Q$, but this coupling not permitted in the superpotential since $\widetilde{Q} X^{*} Q$ is not a chiral operator and therefore its magnetic counterpart cannot appear in the superpotential at all.

Thus we see that in the examples above, the chiral operators obtained in this way from the non-chiral module are products of non-trivial operators such that the product factorizes in the chiral ring. Whether

this is a general feature or not is unclear, nor is the part it may play in the larger picture.

\section{Correspondence with an Arbitrary Kähler Potential}

Since we do not know the effective Kähler potential that should be used in these formulas, we would like to find the analogue of these correspondences for an arbitrary Kähler potential, again taking the classical objects of symbols modulo relations.

The first thing to note is that there is a preferred Kähler potential in this problem, i.e. one can overcome the usually inherent ambiguity associated with Kähler transformations. Given an arbitrary Kähler potential it is natural to define the invariant Kähler potential (obtained by averaging over orbits of all the symmetries in the problem, and particularly the R-symmetry). Once done, then the inherent arbitrariness in Kähler transformations 
$K \rightarrow K+F(X)+F^{*}\left(X^{*}\right)$ is gone since, by unitarity constraints, there is no gauge invariant holomorphic regular function with $\mathrm{R}$ charge 0 .

The generalization of $\tilde{q} D q-(D \tilde{q}) q$ is $O_{\dot{\alpha}}=\frac{\partial K_{m}}{\partial \tilde{q}_{\tilde{k}}^{* i}}\left(\mathbf{D}_{\alpha} \frac{\partial K_{m}}{\partial q_{i l}^{*}}\right)-\left(\mathbf{D}_{\alpha} \frac{\partial K_{m}}{\partial \tilde{q}_{\tilde{k}}^{* i}}\right) \frac{\partial K_{m}}{\partial q_{i l}^{*}}$. Note that the matching of quantum numbers requires that $K_{m}$ be the invariant Kähler potential above. Then $\epsilon^{\dot{\beta} \dot{\alpha}} \overline{\mathbf{D}}_{\dot{\beta}} O_{\dot{\alpha}}=-4\left(\frac{\partial K_{m}}{\partial \tilde{q}_{\tilde{k}}^{* i}} \frac{\partial W_{m}^{*}}{\partial q_{i l}^{*}}-\frac{\partial W_{m}^{*}}{\partial q_{i l}^{*}} \frac{\partial K_{m}}{\partial \tilde{q}_{\dot{k}}^{* i}}\right)$, which can be written in terms of the global symmetry currents. Similarly in the electric theory the $(0,0)$ descendant is still null because $W=0$.

There are two points to be made about this operator. First is that it can be generalized in a trivial way $O_{\dot{\alpha}}^{(P)}=\frac{\partial K_{m}}{\partial \tilde{q}_{\tilde{k}}^{* i}} P(X)\left(\overline{\mathbf{D}}_{\dot{\alpha}} \frac{\partial K_{m}}{\partial q_{i l}^{*}}-\left(\overline{\mathbf{D}}_{\dot{\alpha}} \frac{\partial K_{m}}{\partial \tilde{q}_{\bar{k}}^{* i}}\right) P(X) \frac{\partial K_{m}}{\partial q_{i l}^{*}}\right.$ where $P$ is any holomorphic polynomial in whatever fields we have in the theory.

The second comment regards the geometrical meaning of $O_{\dot{\alpha}, \bar{k} l}$. Let us discuss for a minute a similar object in a WZ model, i.e., the object $\left(\frac{\partial K}{\partial X^{i *}}\left(D_{\alpha} \frac{\partial K}{\partial X^{j *}}\right)-\right.$ $\left.\left(D_{\alpha} \frac{\partial K}{\partial X^{i *}}\right) \frac{\partial K}{\partial X^{j *}}\right) d x^{i *} \wedge d x^{j *}$. This is clearly a closed two form. The transformation law of our object $O$ above is similar but it is not quite a 2 form. We can however write it as the components of the two form $\left(\frac{\partial K_{m}}{\partial \tilde{q}_{\tilde{k}}^{* i}}\left(\overline{\mathbf{D}}_{\dot{\alpha}} \frac{\partial K_{m}}{\partial q_{j l}^{*}}\right)-\left(\overline{\mathbf{D}}_{\dot{\alpha}} \frac{\partial K_{m}}{\partial \tilde{q}_{\tilde{k}}^{* i}}\right) \frac{\partial K_{m}}{\partial q_{j l}^{*}}\right) d q^{j l *} d \tilde{q}^{* i \tilde{k}}$ evaluated on an appropriate set of gauge invariant bi-vectors.

The second object, of the type $\tilde{Q}^{*} \overline{\mathbf{D}} \cdot \mathbf{D} \overline{\mathbf{D}} \cdot Q^{*}$, remains the same in the theory with an arbitrary Kähler potential. The only change is that one has to insert the gauge current appropriate to K. Expression 3.5 remains essentially the same except that every $M$ appearing should be replaced by $\frac{\partial K}{\partial M^{*}}$.

\section{Final Comment}

We would make one final observation. Calculating the $(1,0)$ descendant of both sides of (3.3), one obtains

$$
\widetilde{Q}^{*} F_{\alpha \beta} Q^{*} \leftrightarrow \psi_{\{\alpha}^{m} \tilde{\psi}_{\beta\}}^{m}
$$

where $\psi^{m}\left(\tilde{\psi}^{m}\right)$ are the fermion partners of $q$ and $\tilde{q}$.

The physics behind this expression is unclear. It is intriguing, however, that at the end of the day the field strength (as measured in the LHS) is a fermion matter bi-linear in the dual theory. 


\section{Acknowledgments}

It is a pleasure to thank P. Argyres, T. Banks, M. Peskin and N. Seiberg for useful discussions and comments, and to thank SLAC for its hospitality at the early stages of this work. 


\section{References}

[1] N. Seiberg, Nucl. Phys. B435 (1995) 129.

[2] K. Intriligator, N. Seiberg, RU-95-48, IASSNS-HEP-95/70.

[3] A. B. Zamolodchikov, Sov. J. Nucl. Phys. 44(1986)529-533.

[4] J. Wess, Lecture Notes in Physics 77, Springer, Berlin 1978; P. West, Introduction to Supersymmetry and Supergravity, 2nd ed. World Scientific.

[5] P. Wess, J. Bagger, Supersymmetry and Supergravity, Princeton University Press.

[6] M. A. Luty, W. Taylor IV, MIT-CTP-2440, hep-th/9506098

[7] D. Kutasov, A. Schwimmer, N. Seiberg, EFI-95-68, WIS/95/27, RU-95-75, hepth/9510222.

[8] G. Mack,Comm. Math. Phys. 55 (1977) 1; V.K. Dobrev, V.B. Petkova, Phys. Lett. 162B (1985) 127.

[9] P. Argyres, N. Seiberg, Private Communication.

[10] W. Lerche, C. Vafa, N.P. Warner, Nucl. Phys. B324 (1989) 427.

[11] D. Kutasov, Nucl. Phys. B442 (1995) 447; D. Kutasov, A. Schwimmer, Phys. Lett. 354B (1995) 315.

[12] O. Aharony, J. Sonnenschein, S. Yankielowicz, Nucl. Phys. B449 (1995) 509; K. Intriligator, R.G. Leigh, M.J. Strassler, hep-th/9506148; K. Intriligator, Nucl. Phys. B448 (1995) 187.

[13] T. Banks, A. Zaks, Nucl. Phys. B196 (1982) 189 